\title{
Optimization of the Observer Motion for Bearings-Only Target Motion Analysis ${ }^{1}$
}

\author{
J.P. Le Cadre, IRISA/CNRS \\ Campus de Beaulieu
}

35042 Rennes, France. (e-mail: lecadre@irisa.fr)

\begin{abstract}
This paper deals with the optimization of the receiver trajectory for target motion analysis. The observations are made of estimated bearings. The problem consists in determining the sequence of controls (e.g.: the receiver headings) which maximizes a cost functional. This cost functional is generally a functional of the FIM matrix (Fisher Information Matrix).
\end{abstract}

\section{Introduction}

A fundamental problem for ${ }^{1}$ BOT tracking is the following: if the system is observable what is the accuracy of the state estimate and how to optimize the inputs of the system? In this system approach, the observer maneuvers are the system inputs. This is a very difficult problem of control since, in the first hand, the system is only partially observed, and in the second, the cost functional is non-additive. This means that the effects of the inputs are not separable.

A classic approach consists then in considering the Fisher Information Matrix (FIM) and more precisely its determinant. The choice of the determinant functional is reasonable. However, as we shall see later, the det functional does not have the (additive) monotonicty property, so that the classical tools like the dynamic programming priciple or the Pontryagin Maximum principle are irrelevant.

This explains, for a large part, the relative complexity of this problem. We shall show that using elementary multilinear algebra accurate approximations of $\operatorname{det}$ ( FIM) may be obtained. More specifically, we shall prove that $\operatorname{det}$ ( FIM) may be approximated by a functional involving only the successive source bearingrates, thus yielding the general form of the optimal inputs (observer maneuvers). In particular it will be shown that, under the long-range and bounded controls hypotheses, the sequence of optimal controls lies in the general class of bang-bang controls. These results demonstrate the interest of maneuver diversity.

\footnotetext{
${ }^{1}$ This work has been supported by Direct. Constr. Navales (DCN/Ing), France

${ }^{1}$ BOT means Bearings Only Tracking

$0-7803-3970-8 / 97 \$ 10.00$ () 1997 IEEE
}

\section{Problem formulation}

The source, located at the coordinates $\left(r_{x s}, r_{y s}\right)$ moves with a constant velocity vector $\mathrm{v}\left(v_{x s}, v_{y s}\right)$ and is thus defined to have the state vector $[1,2]$ :

$$
\mathbf{X}_{s} \triangleq\left[r_{x s}, r_{y s}, v_{x s}, v_{y s}\right]^{*}
$$

The observer state is similarly defined as :

$$
\mathbf{X}_{r e c} \triangleq\left[r_{x r e c}, r_{y r e c}, v_{x r e c}, v_{y r e c}\right]^{*} \text {, }
$$

so that, in terms of the relative state vector $\mathbf{X}$ defined by :

$$
\mathbf{X}=\mathbf{X}_{s}-\mathbf{X}_{r e c} \triangleq\left[r_{x}, r_{y}, v_{x}, v_{y}\right]^{*}
$$

the discrete-time equation of the system (i.e. the equation of the relative motion) takes the following form :

$$
\mathbf{X}_{k+1}=F \mathbf{X}_{k}+\mathbf{U}_{k}
$$

where :

$F=\Phi(k, k+1)=\left(\begin{array}{cc}I d_{2} & \alpha I d_{2} \\ 0 & I d_{2}\end{array}\right), I d_{2} \triangleq\left(\begin{array}{ll}1 & 0 \\ 0 & 1\end{array}\right)$

and

$$
\alpha \triangleq t_{k+1}-t_{k}=c s t
$$

In the above formula $t_{k}$ is the time at the $k$-th sample while the vector $\mathrm{U}_{k}=\left(0,0, u_{x}(k), u_{y}(k)\right)^{*}$ accounts for the effects of observer accelerations (or controls). ). The measurements are the estimated angles $\hat{\theta}_{k}$ (bearings) from the observer to the source, so that the observation equation stands as follows:

$$
\hat{\theta}_{k}=\theta_{k}+w_{k}
$$

with :

$$
\theta_{k}=\tan ^{-1}\left(\frac{r_{x}(k)}{r_{y}(k)}\right)
$$

and $w_{k}$ is the measurement noise.

Given the history of measured bearings $\hat{\Theta} \triangleq\left\{\hat{\theta}_{i}\right\}_{i=1}^{n}$ the likelihood function is : 


$$
P(\hat{\boldsymbol{\Theta}} \mid \mathbf{X})=\operatorname{cst} \exp \left[-\frac{1}{2}\|\hat{\boldsymbol{\Theta}}-\boldsymbol{\Theta}(\mathbf{X})\|_{\Sigma}^{2}\right]
$$

$\Theta(\mathbf{X})$ defined by eqs $(2,3)$

and

$$
\underset{\Sigma=\operatorname{diag}\left(\sigma^{2}\right)}{\|\hat{\boldsymbol{\Theta}}-\boldsymbol{\Theta}(\mathbf{X})\|_{\Sigma}^{2} \triangleq(\hat{\boldsymbol{\Theta}}-\boldsymbol{\Theta}(\mathbf{X}))^{*} \Sigma^{-1}(\hat{\boldsymbol{\Theta}}-\boldsymbol{\Theta}(\mathbf{X}))}
$$

The calculation of the gradient vector is easily derived from (4), yielding :

$$
\frac{\partial \Theta(\mathbf{X})}{\partial \mathbf{X}}=\left(\begin{array}{cccc}
\frac{\cos \theta_{1}}{r_{1}} & -\frac{\sin \theta_{1}}{r_{1}} & \frac{\cos \theta_{1}}{r_{1}} & -\frac{\sin \theta_{1}}{r_{1}} \\
\vdots & & & \\
\frac{\cos \theta_{n}}{r_{n}} & -\frac{\sin \theta_{n}}{r_{n}} & \frac{n \cos \theta_{1}}{r_{n}} & -\frac{n \sin \theta_{n}}{r_{n}}
\end{array}\right)
$$

where $\left\{\theta_{i}\right\}_{i=1}^{n}$ represent the source bearing at the instant $i$ and $\left\{r_{i}\right\}$ the source-observer distance. In (5), the reference time is the instant 0 . Obviously, another reference time may be chosen but it is quite remarkable that the determinant of the FIM does not depend on the reference time.

Consider the case of a non maneuvering source (constant velocity vector), then the calculation of the FIM is a routine exercice yielding, under the Gaussian assumption:

$$
\mathrm{FIM}=\left(\frac{\partial \Theta(\mathbf{X})}{\partial \mathbf{X}}\right)^{*} \Sigma^{-1}\left(\frac{\partial \Theta(\mathbf{X})}{\partial \mathbf{X}}\right)
$$

Thus, we deal with the following problem :

Denoting $\mathbf{G}_{k}$ the gradient vector of the log-likelihood functional (5), i.e. :

$\mathbf{G}_{k}=\frac{1}{\sigma_{k} r_{k}}\left(\cos \left(\theta_{k}\right),-\sin \left(\theta_{k}\right), k \cos \left(\theta_{k}\right),-k \sin \left(\theta_{k}\right)\right)^{*}$,

the problem is to determine the sequence of controls $\left\{u_{1}, \cdots, u_{n}\right\}$ (denoted $\mathbf{U}$ ) such that :

$$
\begin{array}{r}
\mathbf{U} \rightarrow \arg \max \left(\operatorname{det}\left(\sum_{k=1}^{n} \mathbf{G}_{k} \mathbf{G}_{k}^{*}\right)\right. \\
\tan \left(\theta_{k}\right)=\frac{r_{x}(0)+k v_{x}+\sum_{l=1}^{k} u_{l, x}}{r_{y}(0)+k v_{y}+\sum_{l=1}^{k} u_{l, y}} .
\end{array}
$$

The difficulty and the originality of the above problem stem from the two following facts. First, the source motion is unknown which means that the state vector $\mathrm{X}$ is unknown.
Second, the effects of the various controls are not separable. In this context, the dynamic programming principle should be very attractive. Unfortunately, it necessarily requires that the cost functional $f$ (from $\mathcal{H}_{n}$, the vector space of $n$-dimensional hermitian matrix to $\mathbb{R}$ ) satisfies the following motonicity property, denoted MDP (Matrix Dynamic Programming Property) and defined below :

\section{Definition 1}

$$
\begin{aligned}
& \text { - } f \text { is smooth }\left(\mathcal{C}^{2}\right) \\
& \text { - let } A \text { and } B \text { in } \mathcal{H}_{n} \\
& \text { be two matrices and assume that: } \\
& \quad f(B)>f(A) \\
& \text { then whatever the matrix } C \text { in } \mathcal{H}_{n} \text {, we have: } \\
& \quad f(B+C)>f(A+C)
\end{aligned}
$$

So, a fundamental question consists in determining the functionals $f$ having the MDP property. An answer is provided with the following result [3].

Property 1: Let $f$ satisfying the MDP property then :

$$
f(A)=g(\operatorname{tr}(A R))
$$

where $g$ is any monotonic increasing function and $R$ is a fixed matrix.

\section{A direct analysis of the FIM determinant :}

For the sake of simplicity, the following assumptions are made along this section. First, the distance will be assumed to be constant. Further, we consider that the diagonal noise matrix: $\Sigma$ is proportional to the identity (i.e. $\Sigma=\sigma^{2} I d$ ). Even if the first hypothesis seems rather restrictive, we shall see later that the effects of range and bearing-rate variations are uncoupled, allowing us to analyze them separately. Furthermore, the effects of range variations are concentrated in a multiplicative term, factor of the determinant.

We shall thus consider a simplified model of the source motion :

$$
\theta_{i+j}=\theta_{i}+j \dot{\theta}+\sum_{k=i+1}^{l} u_{k},
$$

where $\dot{\theta}$ is the bearing-rate (for a given reference time), and $u$ is the bearing-rate change corresponding to an observer maneuver (control). For the sequel, the controls will be the observer bearing-rate changes $u_{k}$.

The fundamental interest of this approach lies in the fact that no a priori knowledge of the source trajectory is assumed.

We shall denote $F_{k_{0}, 4}$ the FIM corresponding to an arbitrary reference time $k_{0}$ and 4 consecutive measurements, $\theta_{k_{0}}, \cdots, \theta_{k_{0}+3}$. Then the FIM $F_{k_{0}, 4}$ takes the 
following form (4 measurements $\left.{ }^{2}\right)$ :

$$
F_{k_{0}, 4}=(\sigma r)^{-2} \mathcal{G}_{k_{0}, 4} \mathcal{G}_{k_{0}, 4}^{*}
$$

where :

$$
\mathcal{G}_{k_{0}, 4}=\left(\mathbf{G}_{k_{0}}, \mathbf{G}_{k_{0}+1}, \mathbf{G}_{k_{0}+2}, \mathbf{G}_{k_{0}+3}\right) .
$$

and $\mathbf{G}_{k}$ is the gradient vector of the observation $\theta_{k}$ w.r.t. $\mathrm{X}_{0}$, i.e. :

$$
\mathbf{G}_{k}=\left(\cos \theta_{k},-\sin \theta_{k}, k \cos \theta_{k},-k \sin \theta_{k}\right)^{*}
$$

Assuming $\mathcal{G}_{k_{0}, 4}$ invertible, we have :

$$
\operatorname{det}\left(F_{k_{0}, 4}\right)=(\sigma r)^{-8}\left(\operatorname{det} \mathcal{G}_{k_{0}, 4}\right)^{2} .
$$

Of course, our attention is not limited to four measurements per legs. So, the previous calculations will now be extended to any number of measurements. Let $\ell$ be the number of measurements and consider now the $(4 \times 4)$ FIM $F_{k, \ell}(l \geq 4)$ defined as in $(9)$ by $^{3}$ :

$$
F_{k_{0}, \ell}=(\sigma r)^{-2} \mathcal{G}_{k_{0}, \ell} \mathcal{G}_{k_{0}, \ell}^{*}
$$

where :

$$
\mathcal{G}_{k_{0}, \ell}=\left(\mathbf{G}_{k_{0}}, \mathbf{G}_{k_{0}+1}, \cdots, \mathbf{G}_{k_{0}+\ell}\right) \quad \ell \geq 0
$$

Using classical properties of multilinear algebra, namely the Cauchy-Binet formula, $\operatorname{det}\left(F_{k_{0}, \ell}\right)$ is given by the following formula :

$$
\operatorname{det}\left(F_{k_{0}, \ell}\right)=(\sigma r)^{-8} \sum_{E}\left[\operatorname{det}\left(\mathcal{G}_{E}\right)\right]^{2}
$$

where :

$$
E=\left\{i_{1}, i_{2}, i_{3}, i_{4}\right\} \text { s.t. } 1 \leq i_{1}<i_{2}<i_{3}<i_{4} \leq \ell
$$

and :

$$
\mathcal{G}_{E}=\left(\mathbf{C}_{i_{1}}, \mathbf{C}_{i_{2}}, \mathbf{C}_{i_{3}}, \mathbf{C}_{i_{4}}\right) \quad \mathbf{C}_{i_{j}} \triangleq \mathbf{G}_{k_{0}+i_{j}} .
$$

We stress that the above formula plays a central role in the analysis of the FIM determinant.

The case of constant bearing-rate :

In (11) $\mathbf{C}_{i_{j}}$ stands for the $i_{j}$-th column of the matrix $\mathcal{G}$. Considering for instance, a first order expansion of the bearings $\theta_{k_{0}+i}$ (i.e. $\theta_{k_{0}+i} \stackrel{1}{=} \theta_{k_{0}}+i \dot{\theta}$ ), the calculation of $\operatorname{det}\left(F_{k_{0}, \ell}\right)$ is reduced to the calculation of the determinants $\operatorname{det}\left(\mathcal{G}_{E}\right)$. Now each of these determinants is the determinant of a $4 \times 4$ matrix. Its calculation is greatly eased by using the following basic result.

\footnotetext{
${ }^{2}$ more generally $F_{k_{0}, l}=(\sigma r)^{-2} \mathcal{G}_{k_{0}, l} \mathcal{G}_{k_{0}, l}^{*}$

${ }^{3}$ Note that the source-observer distance is again assumed to be constant.
}

Property 2: Let $\mathbf{E}$ the first vector of the canonical basis of $\mathbb{R}^{4},\left(\mathbf{E}=(1,0,0,0)^{*}\right)$, then the following equality holds :

$$
\operatorname{det} \mathcal{G}_{E}=\operatorname{det}\left(R_{1}^{i_{1}} \mathbf{E}, R_{1}^{i_{2}} \mathbf{E}, R_{1}^{i_{3}} \mathbf{E}, R_{1}^{i_{4}} \mathbf{E}\right)
$$

Proof :Consider the determinant $\operatorname{det} \mathcal{G}_{E}$ (see eq. 11) where as previously, $E=\left\{i_{1}, i_{2}, i_{3}, i_{4}\right\}$ and $i_{1}<i_{2}<$ $i_{3}<i_{4}$.

$$
\begin{aligned}
\operatorname{det} \mathcal{G}_{E} & =\operatorname{det}\left(\mathbf{G}_{i_{1}}, \cdots, \mathbf{G}_{i_{4}}\right) \\
& =\operatorname{det}\left(R_{1}^{i_{1}} \mathbf{G}_{k_{0}}, R_{1}^{i_{2}} \mathbf{G}_{k_{0}}, R_{1}^{i_{3}} \mathbf{G}_{k_{0}}, R_{1}^{i_{4}} \mathbf{G}_{k_{0}}\right)
\end{aligned}
$$

where :

$$
R_{1} \triangleq\left(\begin{array}{cc}
R_{0} & 0 \\
R_{0} & R_{0}
\end{array}\right) \text { and, } R_{0} \triangleq\left(\begin{array}{cc}
\cos \dot{\theta} & \sin \dot{\theta} \\
-\sin \dot{\theta} & \cos \dot{\theta}
\end{array}\right)
$$

In the same spirit, the vector $\mathbf{G}_{k_{0}}$ may be written as :

$$
\mathbf{G}_{k_{0}}=T_{1}^{k_{0}} \mathbf{E}
$$

where :

$$
\begin{gathered}
T_{1} \triangleq\left(\begin{array}{cc}
T_{0} & 0 \\
T_{0} & T_{0}
\end{array}\right) \text { and }, T_{0} \triangleq\left(\begin{array}{cc}
\cos \theta & +\sin \theta \\
-\sin \theta & \cos \theta
\end{array}\right) \\
\theta \triangleq \theta_{k_{0}} / k_{0}, \quad \mathbf{E}=(1,0,0,0)^{*}
\end{gathered}
$$

Now the following properties are instrumental :

- the matrices $R_{0}$ and $T_{0}$ are rotation matrices, hence they commute ,

$$
\text { - } \operatorname{det}\left(R_{1}\right)=\operatorname{det}\left(R_{0}\right)^{2}=1 \text {. }
$$

The matrices $R_{1}$ and $T_{1}$ then also commute and using this property $\operatorname{det} \mathcal{G}_{E}$ becomes :

$$
\begin{aligned}
\operatorname{det} \mathcal{G}_{E} & \left.=\operatorname{det}\left(T_{1}^{k_{0}} R_{1}^{i_{1}} \mathbf{E}, T_{1}^{k_{0}} R_{1}^{i_{2}} \mathbf{E}, T_{1}^{k_{0}} R_{1}^{i_{3}} \mathbf{E}, T_{1}^{k_{0}} R_{1}^{i_{4}} \mathbf{E}\right) 15\right) \\
& =\operatorname{det}\left(T_{1}^{k_{0}}\right) \operatorname{det}\left(R_{1}^{i_{1}} \mathbf{E}, R_{1}^{i_{2}} \mathbf{E}, R_{1}^{i_{3}} \mathbf{E}, R_{1}^{i_{4}} \mathbf{E}\right) \\
& =\operatorname{det}\left(\mathbf{E}, R_{1}^{i_{2}-i_{1}} \mathbf{E}, R_{1}^{i_{3}-i_{1}} \mathbf{E}, R_{1}^{i_{4}-i_{1}} \mathbf{E}\right)
\end{aligned}
$$

Furthermore, the following property has thus been proved in passing : $\operatorname{det} \mathcal{G}_{E}$ is independent of $k_{0}$ and $\theta_{k_{0}}$. This remarkable property is due to the basic properties of the determinant and the structures of the matrices $R_{1}$ and $T_{1}$.

$\square \square \square$

The above determinant itself (i.e. $: \operatorname{det} \mathcal{G}_{E}=$ $\operatorname{det}\left(\mathbf{E}, R_{1}^{i} \mathbf{E}, R_{1}^{j} \mathbf{E}, R_{1}^{k} \mathbf{E}\right)$ ) can now be easily calculated by means of exterior algebra, yielding the following simple and general result : 
Property 3: $\operatorname{det} \mathcal{G}_{E}=j(k-i) \sin ((k-$ j) $x) \sin (i x)+i(k-j) \sin (j x) \sin ((i-k) x)$,

where : $i=i_{2}-i_{1}, j=i_{3}-i_{1}, k=i_{4}-i_{1}$.

Proof : The calculation of $\operatorname{det} \mathcal{G}_{E}$ is greatly eased by using exterior algebra. The canonical basis of $\mathbb{R}^{4}$ is denoted $\left\{\mathbf{E}_{1}, \cdots, \mathbf{E}_{4}\right\}$. For the coherence of notations, the vector $\mathbf{E}$ is identified with $\mathbf{E}_{1}$. Then, the components of the exterior products $\mathbf{E}_{1} \wedge R_{1}^{i} \mathbf{E}_{1}$, in the "reduced" basis $\left\{\mathbf{E}_{1} \wedge \mathbf{E}_{2}, \mathbf{E}_{1} \wedge \mathbf{E}_{3}, \mathbf{E}_{1} \wedge \mathbf{E}_{4}\right\}$ of $\Lambda^{2}\left(\mathbb{R}^{4}\right)$ are straightforwardly calculated and given below :

$$
\mid \begin{aligned}
& \alpha_{0}=-\sin (i x) \longleftarrow \mathbf{E}_{1} \wedge \mathbf{E}_{2} \\
& \beta_{0}=i \cos (i x) \longleftarrow \mathbf{E}_{1} \wedge \mathbf{E}_{3}, \\
& \gamma_{0}=-i \sin (i x) \longleftarrow \mathbf{E}_{1} \wedge \mathbf{E}_{4} .
\end{aligned}
$$

Similarly, the components of $R_{1}^{j} \mathbf{E}_{1} \wedge R_{1}^{k} \mathbf{E}_{1}$, in the "reduced" basis $\left\{\mathbf{E}_{3} \wedge \mathbf{E}_{4}, \mathbf{E}_{2} \wedge \mathbf{E}_{4}, \mathbf{E}_{2} \wedge \mathbf{E}_{3}\right\}$ are :

$$
\mid \begin{aligned}
& \alpha_{1}=j k \sin ((j-k) x) \longleftarrow \mathbf{E}_{3} \wedge \mathbf{E}_{4}, \\
& \beta_{1}=(k-j) \sin (j x) \sin (k x) \longleftarrow \mathbf{E}_{2} \wedge \mathbf{E}_{4}, \\
& \gamma_{1}=j \sin ((k-j) x)-(k-j) \sin (j x) \longleftarrow \mathbf{E}_{2} \wedge \mathbf{E}_{3} .
\end{aligned}
$$

The determinant $\operatorname{det} \mathcal{G}_{E}$ is deduced from the above calculations, by considering the sum of the coefficients of the vector $\mathbf{E}_{1} \wedge \mathbf{E}_{2} \wedge \mathbf{E}_{3} \wedge \mathbf{E}_{4}$ which spans the 1dimensional space $\Lambda^{4}\left(\mathbb{R}^{4}\right)$, i.e. :

$$
\begin{aligned}
\operatorname{det} \mathcal{G}_{E}= & \alpha_{0} \alpha_{1}-\beta_{0} \beta_{1}+\gamma_{0} \gamma_{1}, \\
= & j(k-i) \sin ((k-j) x) \sin (i x) \\
& +i(k-j) \sin (j x) \sin ((i-k) x) .
\end{aligned}
$$

Using Prop.3 and the Cauchy-Binet formula, a general formulation of $\operatorname{det}(\mathrm{FIM})$ stands as follows :

$$
\begin{array}{r}
\operatorname{det}(\mathrm{FIM})=\sum_{E}(j(k-i) \sin ((k-j) x) \sin (i x), \\
+i(k-j) \sin (j x) \sin ((i-k) x))^{2} .
\end{array}
$$

Practically, the following approximations are easily deduced from the above property .

\section{Result 1 :}

$$
\operatorname{det} \mathcal{G}_{E} \stackrel{6}{=} \frac{(i j k)}{3}(k-i)(k-j)(j-i) x^{4},
$$

and therefore :

$$
\begin{aligned}
& \operatorname{det}\left(F_{k_{0}, l}\right) \approx \\
& \sum_{1<i<j<k \leq l}\left[\frac{(i j k)}{3}(k-i)(k-j)(j-i)\right]^{2}\left(\frac{\sin \dot{\theta}}{\sigma r}\right)^{8} .
\end{aligned}
$$

From Result 1, the following approximation is deduced $^{4}$ :

$$
\begin{aligned}
\operatorname{det}\left(F_{k_{0}, l}\right) \approx & P(l)\left(\frac{\sin \dot{\theta}}{\sigma r}\right)^{8} \alpha^{-1} \\
& \propto \ell^{16}\left(\frac{\sin \dot{\theta}}{\sigma r}\right)^{8}
\end{aligned}
$$

Using the previous formalism, an extension to higher order expansions of $\theta_{k_{0}+i}$ is quite straightforward but not truly enlightening.

\section{Remarks :}

- If a 3-rd order expansion of $\theta_{k_{0}+i}$ is considered in place of the 1-st order one then the value of $\operatorname{det}$ (FIM) is exactly zero. This corroborates the fact that the TMA problem is not observable when the observer does not maneuver.

- However, the BOT problem is observable if multiple measurements are available (at each time). In this case, bounds derived from (20) are accurate.

-In fact, a small variation model for the bearing-rate, i.e. : $\dot{\theta}_{k+1}=\dot{\theta}_{k}+\eta_{k}$ ( $\eta_{k}$ w.g.n.) yields a value of the type (20), eq. 20 roughly appears as an upper bound of $\operatorname{det}($ FIM) .

It has thus been shown that $\operatorname{det}\left(F_{k_{0}, l}\right)$ is proportional to $\ell^{16}\left(\frac{\sin \dot{\theta}}{\sigma r}\right)^{8}$. As practically, $\dot{\theta}$ is very small, this means that $\operatorname{det}\left(F_{k_{0}, l}\right)$ remains very small as far as no observer maneuver occurs. So, we shall now investigate the effects of a bearing-rate change.

\section{The case of bearing-rate change}

We shall now quantify the effects of observer maneuvers. First, the following property is an extension of the previous one to this case.

Consider that the temporal evolutions of the source bearings on two successive legs are described by the two following linear models :

$$
\begin{aligned}
\theta_{k_{0}+i} \stackrel{1}{=} \theta_{k_{0}}+i \dot{\theta}_{1} \text { on the } 1^{-s t} \mathrm{leg} \\
\theta_{k_{0}^{\prime}+j} \stackrel{1}{=} \theta_{k_{o}^{\prime}}+j \dot{\theta}_{2} \text { on the } 2^{-n d} \mathrm{leg} .
\end{aligned}
$$

Then the following property holds (see [2]) and extends the previous result (Prop.3):

Property 4: Consider the case of two consecutive bearing-rates $x$ and $y$, then we have:

$$
\begin{aligned}
& \operatorname{det}\left(\mathbf{E}_{1}, R_{1}^{i} \mathbf{E}_{1}, R_{2}^{j^{\prime}} R_{1}^{i} \mathbf{E}_{1}, R_{2}^{k^{\prime}} R_{1}^{i} \mathbf{E}_{1}\right)= \\
& \left(i+j^{\prime}\right)\left(k^{\prime}\right) \sin (i x) \sin \left(\left(k^{\prime}-j^{\prime}\right) y\right), \\
& -i\left(k^{\prime}-j^{\prime}\right) \sin \left(i x+j^{\prime} y\right) \sin \left(k^{\prime} y\right) .
\end{aligned}
$$

$$
\begin{array}{cc}
{ }^{4} \alpha & 54432000 \\
{\left[\ell^{3}(1+\ell)^{4}\left(\ell^{2}+2 \ell-8\right)\left(\ell^{2}+2 \ell-3\right)^{2}(2+l)^{3}\right]} & P(l)
\end{array}
$$


In (22), the matrices $R_{1}$ and $R_{2}$ are the bearing-rate matrices (cf. 13) associated with the bearing-rates $x$ and $y$. From (22), the following approximations are easily deduced :

$$
\begin{aligned}
& \operatorname{det}\left(\mathbf{E}_{1}, R_{1}^{i} \mathbf{E}_{1}, R_{2}^{j^{\prime}} R_{1}^{i} \mathbf{E}_{1}, R_{2}^{k^{\prime}} R_{1}^{i} \mathbf{E}_{1}\right) \approx \\
& i\left(j^{\prime}\right)\left(k^{\prime}-j^{\prime}\right)\left(k^{\prime}\right) y\left(x-y+\frac{i^{2}}{2} x^{2} y\right) .
\end{aligned}
$$

The proof of Prop. 4 is completely similar to that of Prop. 3. So, we omit it.

The above property allows us to approximate $\operatorname{det} F_{k_{0}, \ell}$ in the case of a maneuvering observer and thus to investigate the effects of the observer maneuvers. In particular, the role of the bearing-rate changes then clearly appears. Indeed, since the parameters $\dot{\theta}_{1}$ and $\dot{\theta}_{2}$ are usually small (both are proportional to $1 / r^{2}$ ), we shall examine an expansion of $\operatorname{det}\left(\mathcal{G}_{E}\right)$ w.r.t. $\dot{\theta}_{1}$ and $\dot{\theta}_{2}$ around the point $(0,0)$. Then, we obtain the following types ${ }^{5}$ of fourth-order expansions (in $\dot{\theta}_{1}$ and $\left.\dot{\theta}_{2}\right)$ of $\operatorname{det}\left(\mathcal{G}_{E}\right)$ :

$$
\begin{aligned}
&\left(\operatorname{det} \mathcal{G}_{E}\right)^{2} \simeq K(y(x-y))^{2}, \\
& \text { or : } K(x(x-y))^{2}
\end{aligned}
$$

with :

$$
K>0, \quad x \triangleq \dot{\theta}_{1}, y \triangleq \dot{\theta}_{2} .
$$

This result is quite fundamental for TMA and will be clarified by a geometric interpretation. For the two bearing-rate case, the expansion of $\operatorname{det} F_{k_{0}, \ell_{1}, \ell_{2}}{ }^{6}$ is :

$$
\operatorname{det} F_{k_{0}, \ell_{1}, \ell_{2}} \simeq \frac{1}{(\sigma r)^{8}}\left[\sum_{i=1}^{5} P_{i}\left(\ell_{1}, \ell_{2}\right) y^{5-i} x^{i-1}\right]
$$

where the polynomials $\left\{P_{i}\left(\ell_{1}, \ell_{2}\right)\right\}_{i=1}^{5}$ are detailed in [3].

From (25), we note that the maximum value of $\operatorname{det} F_{k_{0}, \ell_{1}, \ell_{2}}$ is proportional to $\ell^{12} \dot{\theta}^{4}\left(\ell_{1} \simeq \ell_{2}, \dot{\theta}_{1} \simeq\right.$ $\left.-\dot{\theta_{2}}\right)$.

In fact, denoting $F_{\ell}(x)$ the FIM associated with a constant bearing-rate $x$ and $F_{\ell / 2, \ell / 2}(x,-x)$ the FIM associated with a two-leg observer trajectory (leg 1: $\ell / 2$ meas., bear. rate $x$; leg $2: \ell / 2$ meas., bear. rate $-x$ ), we have :

Res. 2:

$$
\begin{aligned}
\frac{\operatorname{det}\left(F_{\ell}(x)\right)}{\operatorname{det}\left(F_{\ell / 2, \ell / 2}(x,-x)\right.} & \simeq \frac{1}{134} \ell^{4} x^{4} \\
& \simeq \frac{1}{134}(\Delta x)(26)
\end{aligned}
$$

where $\Delta x$ denotes the total bearing variation (i.e. $\Delta x=\ell x)$. For usual scenarios, $\Delta x$ is small in regard to 1 and, therefore, the increase in the FIM determinant

\footnotetext{
${ }^{5}$ the type of the expansion only depends on the relative values of $i_{1}, i_{2}, i_{3}, i_{4}$

${ }^{6} \ell_{i}$ measurements asociated with $\dot{\theta}_{i}, i=1,2$
}

gained by optimized observer maneuvers may be rather impressive. Further, note that this gain is proportional to $(\Delta x)^{-4}$. The above calculation is easily extended to the case of a maneuvering source. The dimension of the state vector is then equal to 6 , while the gain of a bearing-rate change is, this time, proportional to $(\Delta x)^{-8}$

Actually, it seems that the optimum corresponds to a "long" first leg in order to maximize the observer baseline, followed by a "short" second leg.

The previous results are more formally summarized by the following property.

Property 5: Let $x_{1}, x_{2}, \cdots, x_{n}$, the consecutive bearing-rates, then :

$$
\begin{aligned}
\arg _{x_{1}, \cdots, x_{n}} \max \operatorname{det}(\mathrm{FIM}) & =\varepsilon\left(\dot{\theta}_{\max },-\dot{\theta}_{\max }, \dot{\theta}_{\max }, \cdots\right) \\
\varepsilon & = \pm 1 .
\end{aligned}
$$

We refer to [3] for a proof of Prop. 5.

\section{Geometric interpretations of the properties of} the FIM determinant

Since we are especially interested in the effects of observer maneuvers, we shall investigate them by means of the previous results and differential calculus. Consider for instance the following determinant $\left(\mathbf{E}=\mathbf{E}_{1}\right)$ :

$$
f(y)=\operatorname{det}\left(\mathbf{E}, R_{1, x}^{i} \mathbf{E}, R_{1, x}^{j} \mathbf{E}, R_{1, x}^{k} R_{1, y}^{l} \mathbf{E}\right),
$$

where:

$x=\dot{\theta}_{1}, y=\dot{\theta}_{2}$.

Let us now calculate the partial derivative $\frac{\partial f}{\partial y}(x)$, we obtain:

$$
\frac{\partial f}{\partial y}(x)=l \operatorname{det}\left(\mathbf{E}, R_{1, x}^{i} \mathbf{E}, R_{1, x}^{j} \mathbf{E}, R_{1, x}^{k+l-1} S_{1, x} \mathbf{E}\right)
$$

where $S_{1, x}=\left(\frac{\partial}{\partial y} R_{1, y}\right)_{(y=x)}$, or, explicitely:

$$
S_{1, x}=\left(\begin{array}{cc}
S_{0, x} & 0 \\
S_{0, x} & S_{0, x}
\end{array}\right)
$$

with:

$$
S_{0, x}=\left(\begin{array}{cc}
-\sin x & -\cos x \\
\cos x & -\sin x
\end{array}\right)
$$

Using the definitions of $R_{1, x}$ and $S_{1, x}$, and denoting $J$ the two-dimensional $\pi / 2$ rotation matrix, the following properties are then easily proved:

Property 6: The following properties hold:

$$
\begin{aligned}
& S_{0, x}=J R_{0, x}, \frac{\partial}{\partial x} S_{0, x}=-R_{0, x}=J^{2} R_{0, x}, \\
& R_{1, x}^{k} S_{1, x}^{k^{\prime}}=S_{1, x}^{k^{\prime}} R_{1, x}^{k}=S_{1, x}^{k+k^{\prime}}, R_{1, x}^{k} S_{1, y}^{k^{\prime}}=S_{1, x+y}^{k+k^{\prime}} .
\end{aligned}
$$


From Prop. 6, we directly deduce :

$$
\begin{aligned}
& \frac{\partial f}{\partial y}(x)=l \operatorname{det}\left(\mathbf{E}, R_{1, x}^{i} \mathbf{E}, R_{1, x}^{j} \mathbf{E}, S_{1,(k+l) x} \mathbf{E}\right), \\
& \frac{\partial^{2} f}{\partial y^{2}}(x, x)=-l^{2} \operatorname{det}\left(\mathbf{E}, R_{1, x}^{i} \mathbf{E}, R_{1, x}^{j} \mathbf{E}, R_{1, x}^{k+l} \mathbf{E}\right), \\
& \text { etc. }
\end{aligned}
$$

At

this

point, it is worth noting that the vector $S_{1, m x} \mathrm{E}=$ $(-\sin m x, \cos m x,-m \sin m x, m \cos m x)^{*}$ is approximately orthogonal to the vectors $\left\{\mathbf{E}, R_{1, x}^{i} \mathbf{E}, R_{1, x}^{j} \mathbf{E}\right\}$. This is due to the fact that $S_{0, x}$ is a $2 \mathrm{D}$-rotation with an angle equal to $x-\pi / 2$. This fact is typical of a fourdimensional state vector and corresponds to a diversity in maneuvers. Thus, $\frac{\partial f}{\partial y}(x)$ is proportional to $x$, while $\frac{\partial^{2} f}{\partial y^{2}}(x, x)$ is proportional to $x^{4}$.

Using the previous calculations, the following approximations are easily proved :

$$
\begin{aligned}
& \frac{\partial f}{\partial y}(x) \approx \alpha x \text { and }: \frac{\partial^{2} f}{\partial y^{2}}(x, x) \approx \beta x^{4}, \\
& \text { so that : } \\
& f(y) \approx \gamma x(y-x) .
\end{aligned}
$$

From (33) it is clear that the increase of $\operatorname{det}\left(F_{x, y}\right)$ is maximized when the terms $(x(x-y))^{2}$ are maximized. Since $\dot{\theta}$ is bounded, an optimal sequence of controls is necessarily a bang-bang one .

Geometric considerations provide also another interpretation of Prop. 5. More precisely, defining $f(x, y)=$ $\operatorname{det}\left(\mathbf{E}, R_{1, x} \mathbf{E}, R_{1, x} R_{1, y} \mathbf{E}, R_{1, x} R_{1, y}^{2} \mathbf{E}\right)$, we see that $\frac{\partial f}{\partial x}$ and $\frac{\partial f}{\partial y}$ are simultaneously null iff :

$$
\begin{aligned}
& \operatorname{det}\left(\mathbf{E}, S_{1, x} \mathbf{E}, R_{1, x} R_{1, y} \mathbf{E}, R_{1, x} R_{1, y}^{2} \mathbf{E}\right) \\
& =\operatorname{det}\left(\mathbf{E}, R_{1, x} \mathbf{E}, R_{\mathbf{1}, x} R_{1, y} \mathbf{E}, R_{1, x} R_{1, y} S_{1, y} \mathbf{E}\right) .
\end{aligned}
$$

It is then easily seen that the maximum of $f^{2}$ is necessarily attained for an extreme point of the constraint set.

This optimization problem may be connected with the Hadamard inequality . Indeed, let us recall a classical formulation of the Hadamard inequality:

$$
\mid \begin{aligned}
& \operatorname{Max}_{\left\{\mathbf{V}_{i}\right\}_{i=1}^{n}}\left|\operatorname{det}\left(\mathbf{V}_{1}, \cdots, \mathbf{V}_{n}\right)\right| \\
& \text { under the constraints }\left\|\mathbf{V}_{1}\right\|=\varepsilon_{1}, \cdots,\left\|\mathbf{V}_{n}\right\|=\varepsilon_{n} \\
& \operatorname{dim}(\mathbf{V})=n .
\end{aligned}
$$

A solution to the above problem is easily obtained by means of differential calculus (Lagrange multipliers) yielding: the vectors solving (35) are mutually orthogonal vectors satisfying the constraints. However, the problem is greatly complicated, here, by the constraints associated with the vectors $\left\{\mathbf{V}_{i}\right\}_{i}$.
Since the values of $\left(-\dot{\theta}_{\max }, \dot{\theta}_{\max }\right)$ can be estimated by the observer (e.g. from the estimated bearings), it remains to determine the optimal number of switchings (from $\dot{\theta}_{\max }$ to $-\dot{\theta}_{\text {max }}$ ) as well as their locations. However, it seems rather impossible to derive a general bound relative to the number of switching.

\subsection{The effects of range variations:}

Up to now, the effects of range variations have not been considered. However, the analysis is greatly simplified if we remark that the effects of range and bearing-rate variations are geometrically uncoupled. This follows easily by considering $\operatorname{det}\left(\mathcal{G}_{E}\right)$. Including the range, the elementary determinant $\operatorname{det}\left(\mathcal{G}_{E}\right)$ becomes :

$$
\begin{aligned}
\operatorname{det} \mathcal{G}_{E} & =\operatorname{det}\left(\frac{1}{r_{i_{1}}} R_{1}^{i_{1}} \mathbf{E}, \cdots, \frac{1}{r_{i_{4}}} R_{1}^{i_{4}} \mathbf{E}\right) \\
& =\frac{1}{r_{i_{1}}} \cdots \frac{1}{r_{i_{4}}} \operatorname{det}\left(R_{1}^{i_{1}} \mathbf{E}, \cdots, R_{1}^{i_{4}} \mathbf{E}\right)
\end{aligned}
$$

so that :

$\operatorname{det}\left(F_{k, \ell}\right)=(\sigma r)^{-8} \sum_{E}\left[\frac{1}{r_{i_{1}}} \cdots \frac{1}{r_{i_{4}}} \operatorname{det}\left(R_{1}^{i_{1}} \mathbf{E}, \cdots, R_{1}^{i_{4}} \mathbf{E}\right)\right]^{2}$

From the above equality, we note that the effects of range and bearing-rate variations are uncoupled.

\section{Conclusion}

Optimization of the observer maneuvers has been considered along this paper. This problem is not relevant of classical optimal control. Using basic tools of multilinear algebra, it has been proved that this functional may be accurately approximated by a functional involving only the successive source bearing-range rates. In particular, it has been shown that under the long-range and bounded controls hypotheses, the sequence of optimal control lies in the general class of bang-bang controls ${ }^{7}$.

\section{References}

[1] S.E. HAMMEL, Optimal Observer Motion for Bearings-Only Localization and Tracking, Ph. D (Applied Math.), Univ. of Rhode Island, 1988.

[2] J.P. LE CADRE and C. JAUfFret, Discrete-Time Observability and Estimability Analysis for Bearings-Only Target Motion Analysis. IEEE Trans. on AES, vol. AES-33, n. 1,Jan. 1997, pp. 178-201.

[3] J.P. Le CADre and S. Laurent-Michel, Optimizing the Receiver Manieuvers for Bearings-Only Tracking, submitted to Automatica, Aug. 1996.

\footnotetext{
${ }^{7}$ We refer to $[3]$ for simulation results
} 